ARTIFICIAL SATELLITES, Vol. 46, No. 3 - 2011

DOI: 10.2478/v10018-012-0002-1

\title{
USING THE INTEGER DECORRELATION PROCEDURE TO INCREASE OF THE EFFICIENCY OF THE MAF A METHOD
}

\author{
S. Cellmer \\ Institute of Geodesy \\ University of Warmia and Mazury in Olsztyn, Olsztyn \\ e-mail: slawomir.cellmer@uwm.edu.pl
}

\begin{abstract}
The Modified Ambiguity Function Approach (MAFA) is a method of GNSS carrier phase processing. In this method, the functional model of the adjustment problem contains the conditions ensuring the "integerness" of the ambiguities. These conditions are expressed in the form of differentiable function. A prerequisite for obtaining the correct solution is a mechanism ensuring not only the "integerness" of the ambiguity but also appropriate localization of the search space in the place where the ambiguities have correct values. One of such mechanisms is cascade adjustment, applying the linear combinations of the signals L1 and L2 with the integer coefficients and various wavelengths. This paper presents another, independent from the previous, approach to increase the efficiency of the MAFA method. It is based on the application of the integer decorrelation matrix to transform observation equations into equivalent, but better conditioned, observation equations. The transformation matrix is obtained in the well-known ambiguity variance-covariance matrix integer decorrelation process.
\end{abstract}

Keywords: GNSS data processing, ambiguity function, MAFA method.

\section{INTRODUCTION}

The main subject of this paper is an improved algorithm of the MAFA (Modified Ambiguity Function Approach) method, Cellmer et al. (2010), Cellmer (2011a), Cellmer (2011b), Cellmer et al. (2011). The MAFA algorithm takes into account the integer nature of the selected parameters without the necessity for the additional stage of the integer search. It is based on the Least-Squares Adjustment (LSA) algorithm with condition equations, in the functional model. In this paper, a new technique to improve the efficiency of the MAFA method is proposed. This technique exploits an integer decorrelation procedure. After transformation of the observation equations with an integer decorrelation matrix, a model of an adjustment problem turns into an equivalent model,although better conditioned.

The next section presents the theoretical basis of the MAFA method. In the third section, the improvement of the efficiency using integer decorrelation procedure is proposed. The last part of the paper gives a numerical example and some conclusions. 


\section{MAFA METHOD}

The following simple form of the observation equation for double differenced (DD) carrier phase observable is assumed, Hofmann-Wellenhof et.al. (2008), Leick (2004), Teunissen (1998):

where:

$$
\Phi+\mathrm{v}=\frac{1}{\lambda} \rho \mathrm{X}_{\mathrm{c}}+\mathrm{N}
$$

$\Phi \quad-$ DD carrier phase observable (in cycles)

$\lambda \quad$ - signal wave length

$\mathrm{V}-$ residual (measurement noise)

$\mathrm{X}_{\mathrm{c}}$ - receiver coordinate vector

$\rho\left(X_{c}\right)$ - DD geometrical range

$\mathrm{N}$ - integer number of cycles (DD initial ambiguity)

The nominal accuracy of the carrier phase measurement is about 0.01 cycle, HofmannWellenhof et al. (2008). Thus, the residual values should be much lower than half a cycle. Hence, taking into account the integer nature of the ambiguity parameter $\mathrm{N}$, the equation (1) can be rewritten in the following form:

$$
\Phi+\mathrm{v}-\frac{1}{\lambda} \rho=\text { round } \Phi-\frac{1}{\lambda} \rho
$$

or

$$
\mathrm{v}=\text { round } \Phi-\frac{1}{\lambda} \rho-\Phi-\frac{1}{\lambda} \rho
$$

where round is a function of rounding to the nearest integer value. The residual (3) does not contain a term $\mathrm{N}$. Nevertheless, it takes into account the integer nature of ambiguities. The right side of the equation (3) can be expressed in the form of the following, differentiable and continuous function, Cellmer and Wielgosz, (2011):

$$
\Psi=\operatorname{round}(\mathrm{s})-\mathrm{s}=\left\{\begin{array}{c}
-\frac{1}{\pi} \arcsin [\sin (\pi \mathrm{s})] \text { for } \mathrm{s} \in \mathrm{s}: \cos (\pi \mathrm{s}) \geq 0 \\
\frac{1}{\pi} \arcsin [\sin (\pi \mathrm{s})] \text { for } \mathrm{s} \in \mathrm{s}: \cos (\pi \mathrm{s})<0
\end{array}\right.
$$

where $\mathrm{s}$ is an auxiliary variable:

$$
\mathrm{s}=\Phi-\frac{1}{\lambda} \rho \text {. }
$$

Each of the DD carrier phase observations is linearized. After linearization, the general formula of the residual equations can be shown in the following form:

$$
\underline{\mathrm{V}}=-\underline{\mathrm{AX}}+\underline{\Delta}
$$

with:

$$
\begin{aligned}
& \underline{A}=\left[\begin{array}{ccc}
\frac{\partial \rho_{1}}{\partial x} & \frac{\partial \rho_{1}}{\partial y} & \frac{\partial \rho_{1}}{\partial z} \\
\frac{\partial \rho_{2}}{\partial x} & \frac{\partial \rho_{2}}{\partial y} & \frac{\partial \rho_{2}}{\partial z} \\
\vdots & \vdots & \vdots \\
\frac{\partial \rho_{n}}{\partial x} & \frac{\partial \rho_{n}}{\partial y} & \frac{\partial \rho_{n}}{\partial z}
\end{array}\right] \\
& \underline{\Delta}=\text { round } \underline{\Phi}-\frac{1}{\lambda} \underline{\rho}-\underline{\Phi}-\frac{1}{\lambda} \underline{\rho}
\end{aligned}
$$

where:

$\underline{\mathrm{V}}-$ residual vector $(\mathrm{n} \times 1)$, 
$\underline{\mathrm{X}}$ - parameter vector (increments to a prioricoordinates vector $\mathrm{X}_{0}$ ),

$\underline{\text { A }}-$ design matrix $(\mathrm{n} \times 3)$,

$\underline{\Delta}-$ misclosures vector $(\mathrm{n} \times 1)$,

The LS solution of the formula (6) is:

$$
\underline{\mathrm{X}}=-\lambda\left(\underline{\mathrm{A}}^{\mathrm{T}} \underline{\mathrm{PA}}\right)^{-1} \underline{\mathrm{A}}^{\mathrm{T}} \underline{\mathrm{P} \Delta},
$$

with $P$ as the weight matrix.

In order to assure the convergence of the computational process to the correct solution, different linear combinations (LC) of L1 and L2 GPS carrier phase observables with integer ambiguities and longer wavelengths are applied in the cascade adjustment (Jung and Enge, 2000). Table 1 presents the linear combinations used in the MAFA method, along with their wavelengths (Han and Rizos 1996; Cellmer et al., 2010).

Table 1 Linear combinations of L1 and L2 signals with integer ambiguity

\begin{tabular}{|c|c|}
\hline $\mathbf{L C}$ & $\boldsymbol{\lambda}[\mathbf{m}]$ \\
\hline$-3 \mathrm{~L} 1+4 \mathrm{~L} 2$ & 1.6281 \\
\hline $\mathrm{L} 1-\mathrm{L} 2$ & 0.8619 \\
\hline $\mathrm{L} 1$ & 0.1903 \\
\hline
\end{tabular}

The above linear combinations were chosen based on the analyses of the theoretical properties of these combinations, Han and Rizos (1996), Urquhart (2009), Cocard and Geiger (1992). The computations are performed successively for different LC in the order listed in Table 1, namely, starting from LC with the longest wavelength and finishing with L1 signal only.

\section{IMPROVEMENT OF THE EFFICIENCY USING THE DECORRELATION PROCEDURE}

The ambiguities $\mathrm{N}$ are usually strongly correlated. Hence, fixing one value of ambiguity through rounding value s in (5) to the nearest integer as in (2), has an impact on the rest of the ambiguities. Therefore, the correlation between ambiguities should be taken into account at rounding the right side of the equation (2). The alternative way of solving this problem can be transformation of the observation equations into the equivalent form but without correlation. This method is proposed here.

Let us assume that $\mathrm{Z}$ is the integer decorrelation matrix, Chang X-W et.al. (2005), Glenn and Svedensen (2006), Jonge and Tiberius (1996), Liu et. al. (1999), Teunissen (1995):

$$
\underline{\mathrm{Q}}_{\mathrm{NZ}}=\underline{\mathrm{ZQ}}_{\mathrm{N}} \underline{\mathrm{Z}}^{\mathrm{T}},
$$

where:

$\underline{Q}_{N}-$ ambiguity covariance matrix

$\mathrm{Q}_{\mathrm{Nz}}-$ diagonal transformed ambiguity covariance matrix.

If $\underline{Z}$ is integer matrix then transformed ambiguity vector, $\underline{N}_{Z}=\underline{Z N}$ must be integer as well.

By multiplying the equation (1) with $\underline{Z}_{2}$ one can obtain a new equation with a new integer ambiguity vector $\mathrm{N}_{\mathrm{z}}$ : 


$$
\underline{\Phi}_{Z}+\underline{V}_{Z}=\frac{1}{\lambda} \underline{\rho}_{Z}\left(\underline{X}_{C}\right)+\underline{N}_{Z}
$$

The free term vector $\underline{\Delta}$ is computed in this case as follows:

$$
\underline{\Delta}_{Z}=\text { round } \underline{\Phi}_{Z}-\frac{1}{\lambda} \underline{\rho}_{Z}-\underline{\Phi}_{Z}-\frac{1}{\lambda} \underline{\rho}_{Z} \text {, }
$$

Thus, the LS solution is obtained:

$$
\mathrm{X}=-\lambda \quad \mathrm{A}_{Z}^{\mathrm{T}} \mathrm{PA}_{\mathrm{Z}}{ }^{-1} \mathrm{~A}_{\mathrm{Z}}^{\mathrm{T}} \mathrm{P} \Delta_{Z}
$$

with:

$$
\underline{\mathrm{A}_{\mathrm{Z}}}=\underline{\mathrm{ZA}}
$$

There are many various methods of finding the decorrelation matrix Z, Hassibi A. and Boyd S. (1998), Jonge and Tiberius (1996), Liu L.T. et. al. (1999), Xu PL (2001). In any case, the ambiguity covariance matrix $Q_{N}$ is required to find the matrix $Z$. The matrix $Q_{N}$ can be evaluated on the basis of the system of observation equations (1) after linearization:

$$
\underline{\mathrm{V}}=\underline{\mathrm{AX}}+\underline{\mathrm{BN}}-\underline{\mathrm{L}}
$$

where:

$\underline{\mathrm{L}}-$ free term (observed minus computed) vector

$\underline{\mathrm{B}}$ - ambiguity functional model matrix

The covariance matrix of the unknown vector $\mathrm{X}_{\Omega}=[\mathrm{X}, \mathrm{N}]^{\mathrm{T}}$ can be presented as:

$$
\mathrm{C}_{\mathrm{x}_{\Omega}}=\left[\begin{array}{cc}
\mathrm{A}^{\mathrm{T}} \mathrm{PA} & \mathrm{A}^{\mathrm{T}} \mathrm{PB} \\
\mathrm{B}^{\mathrm{T}} \mathrm{PA} & \mathrm{B}^{\mathrm{T}} \mathrm{PB}
\end{array}\right]^{-1}=\left[\begin{array}{cc}
\mathrm{Q}_{\mathrm{X}} & \mathrm{Q}_{\mathrm{XN}} \\
\mathrm{Q}_{\mathrm{NX}} & \mathrm{Q}_{\mathrm{N}}
\end{array}\right],
$$

where:

$$
Q_{N}=\left[\begin{array}{llll}
B^{T} P B-B^{T} P A & A^{T} P A & A^{-1} P B
\end{array}\right]^{-1} \text {. }
$$

In the case of the single epoch data, matrix $\mathrm{B}$ is identity and $\mathrm{Q}_{\mathrm{N}}$ computed according to formula (17), is not positive definite. It causes difficulties with the decorrelation procedure and leads to incorrect solutions. Therefore, an additional coefficient $\mathrm{k}$ is imposed, Cellmer (2011):

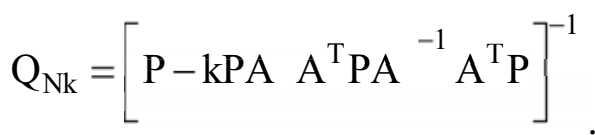

If the value of this coefficient is less than one, it simulates additional observation data (e.g. pseudoranges). It is proposed here to assume $\mathrm{k}=0.99$. This value was derived as optimal from tests. The positive definite matrix $\mathrm{Q}_{\mathrm{Nk}}$ is a biased estimator of the ambiguity covariance matrix. Nevertheless, it is a good approximation for the purpose of decorrelation. The formula (18) can also be used for other than single epoch solutions. When using formula (18) in the case of multi epoch solutions, the decorrelation process is performed separately in each epoch. All decorrelated observation equations are arranged in the form of one observation equation system which is the basis of the LS solution. 


\section{NUMERICAL EXAMPLE}

Tha data come from campaign performed in order to monitor local deformation in open-pit mine „Adamów” in Central Poland. This project is managed by Dr. Radoslaw Baryla from Chair of Satellite Geodesy and Navigation of University of Warmia and Mazury in Olsztyn. Figure 1 depicts the location of the surveys. Two GPS stations of ASG-EUPOS (Polish part of European Positioning System active geodetic network) were used in test surveys ("KONI" and "KUTN"). The surveys were performed on December 9th, 2008, on $50 \mathrm{~km}, 30 \mathrm{~km}$ and 10 $\mathrm{km}$ baselines, with a 30 -second sampling rate. Data sets of each baseline were divided into several dozen (respectively: 82, 98 and 62) 20-minute sessions (40 epochs).

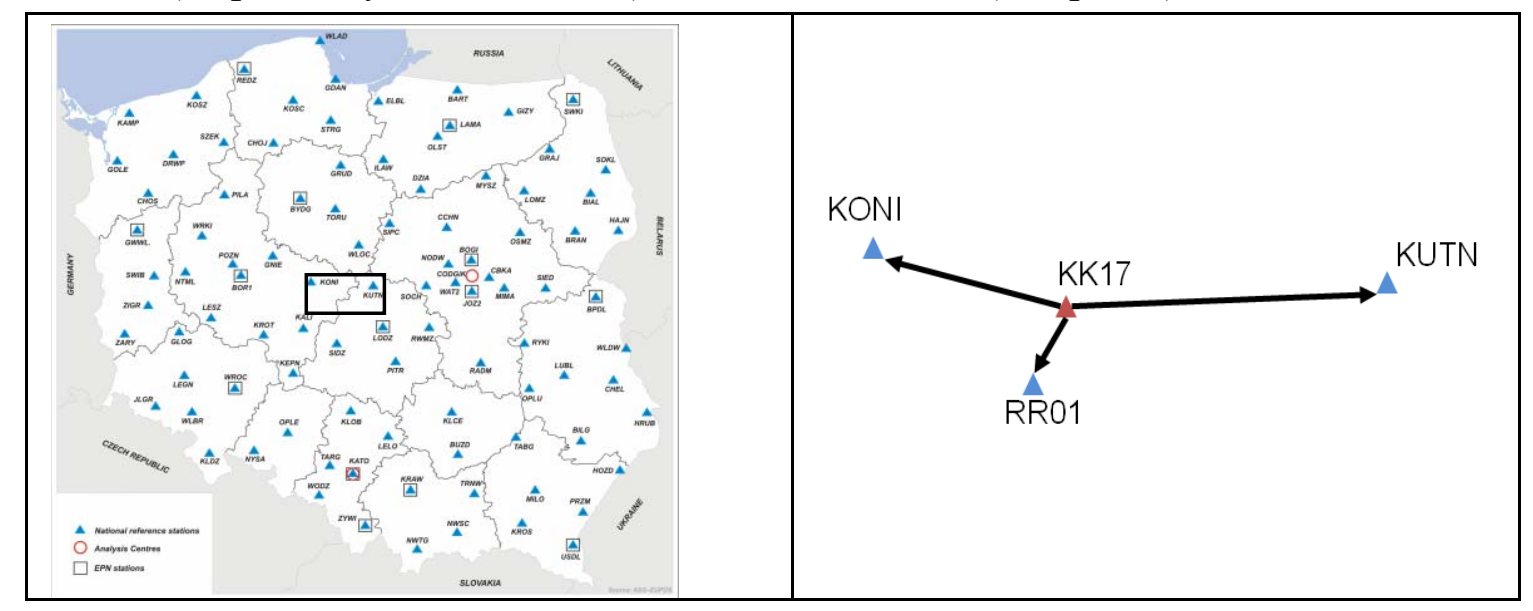

Fig. 1 The location of the test surveys

http://www.asgeupos.pl/webpg/graph/dwnld/map_pl_EN.jpg

The sessions were processed according to proposed approach. In each 20-minute session, the first solution was obtained from one epoch. Further solutions were obtained on the basis of data sets subsequently increased with data from consecutive epochs. The decorrelation procedure was performed in each epoch using the method derived by Liu et.al. (1999). The ambiguity covariance matrix was formed according to formula (18), as a base for the decorrelation procedure. The "true" coordinates were derived using Bernese software on the basis of an 8 hour data set, Dach (2007).

Figure 2 presents the results of some selected sessions as the example from among 180 sessions of the processing of $50 \mathrm{~km}$ and $30 \mathrm{~km}$ baselines. There are linear residuals, with respect to the "true" position from Bernese. The residuals were computed as: $\mathrm{r}=\sqrt{\mathrm{dN}^{2}+\mathrm{dE}^{2}+\mathrm{dU}^{2}}$, where $\mathrm{dN}, \mathrm{dE}, \mathrm{dU}$ are accordingly north, east and vertical components of the residuals with respect to the "true" position.

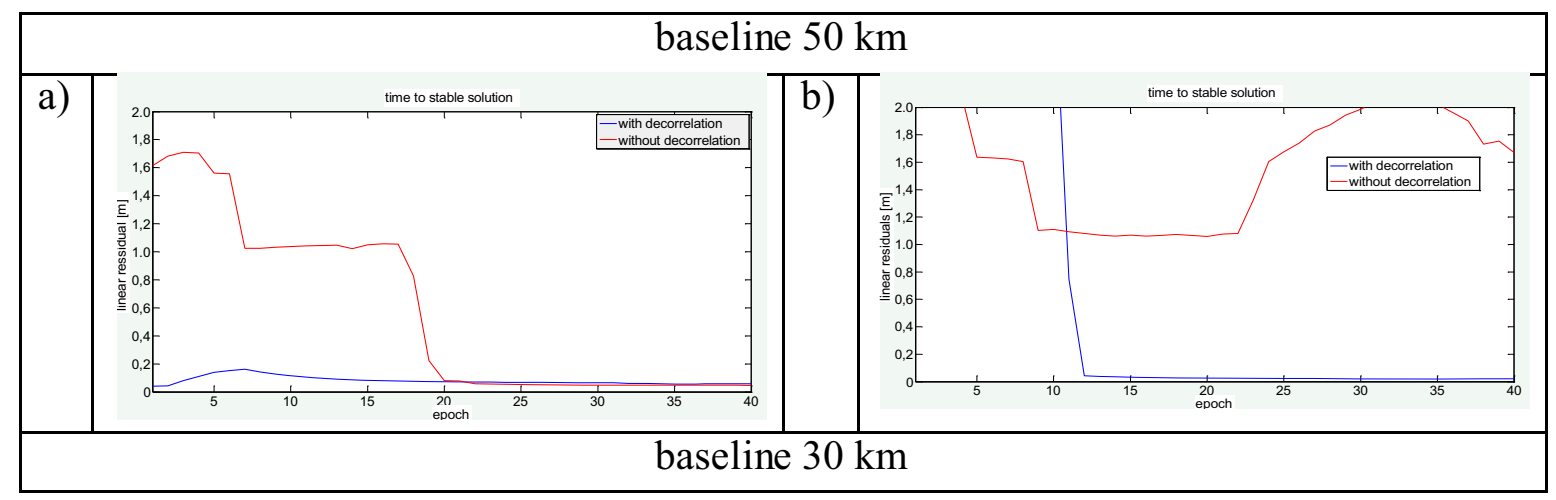




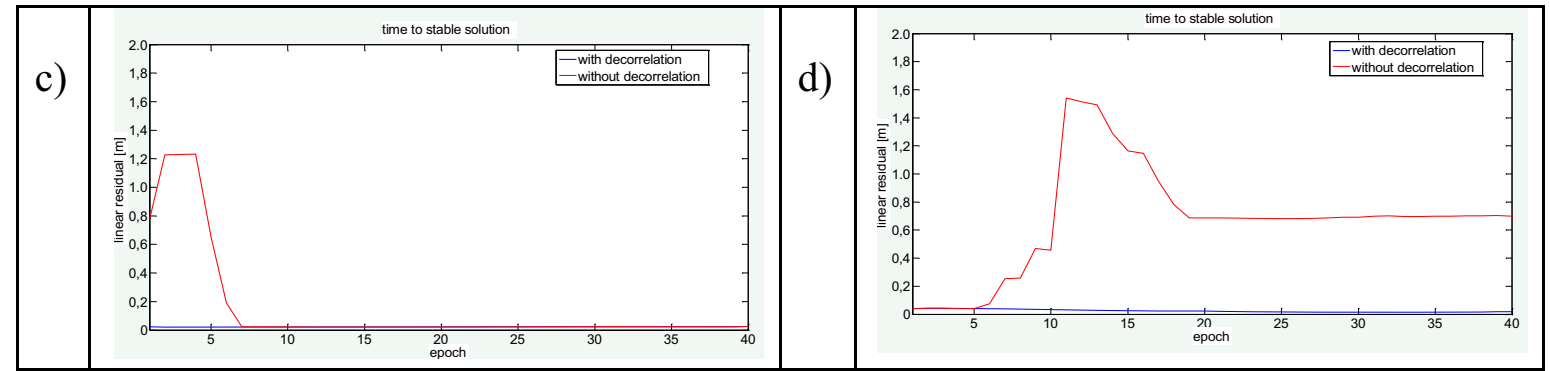

Fig. 2 The results of the baseline processing (selected examples)

The blue line depicts the residuals for the solutions with decorrelation, while the red line represents the solutions without decorrelation. In case a) the solution without decorrelation stabilized after $20^{\text {th }}$ session whereas the solution with decorrelation was close to "true" from the first epoch.

The plot b) presents the session in which the solution without decorrelation gave false results whereas a good solution with decorrelation was obtained in the $12^{\text {th }}$ epoch.

The plots c) and d) show the examples of the results for shorter baseline: about $30 \mathrm{~km}$.

In the session presented in plot c) the solution without decorrelation stabilized after 7th epoch, whereas the correct solution with decorrelation was already stabilized from the 1st epoch. In the case shown in plot d) the solution with decorrelation was correct from 1st epoch and the solution without decorrelation stabilized after 20th epoch on incorrect value $(70 \mathrm{~cm}$ from correct position).

Fig. 3 shows the histograms of the correct stable solutions i.e. the frequency of stabilizing of the correct solution in a respective period of time. The frequency is expressed in [\%] of the total number of the solutions. The histograms were plotted on the basis of the results from 62 sessions for $10 \mathrm{~km}$ baseline, 98 sessions for $30 \mathrm{~km}$ baseline and 82 sessions for $50 \mathrm{~km}$ baseline. As we can see in the first histogram for short baselines, the number of the correct solutions stabilized in respective periods of time is similar for two variants: with and without decorrelation. However for longer baselines, in this case $30 \mathrm{~km}$ (the second column), the number of correct solution obtained in the starting 5 epochs for the variant without decorrelation amounted to $35 \%$ and for the variant with decorrelation almost $70 \%$. The impact of the decorrelation procedure is in this case significant. An even more significant impact of decorrelation procedure occurs for $50 \mathrm{~km}$ baselines (the third column). In the starting 5 epochs, over $60 \%$ correct solutions were obtained for decorrelation variant and less than $5 \%$ for the variant without decorrelation. In over $70 \%$ cases there were no correct solutions until the $40^{\text {th }}$ epoch for the variant without decorrelation.

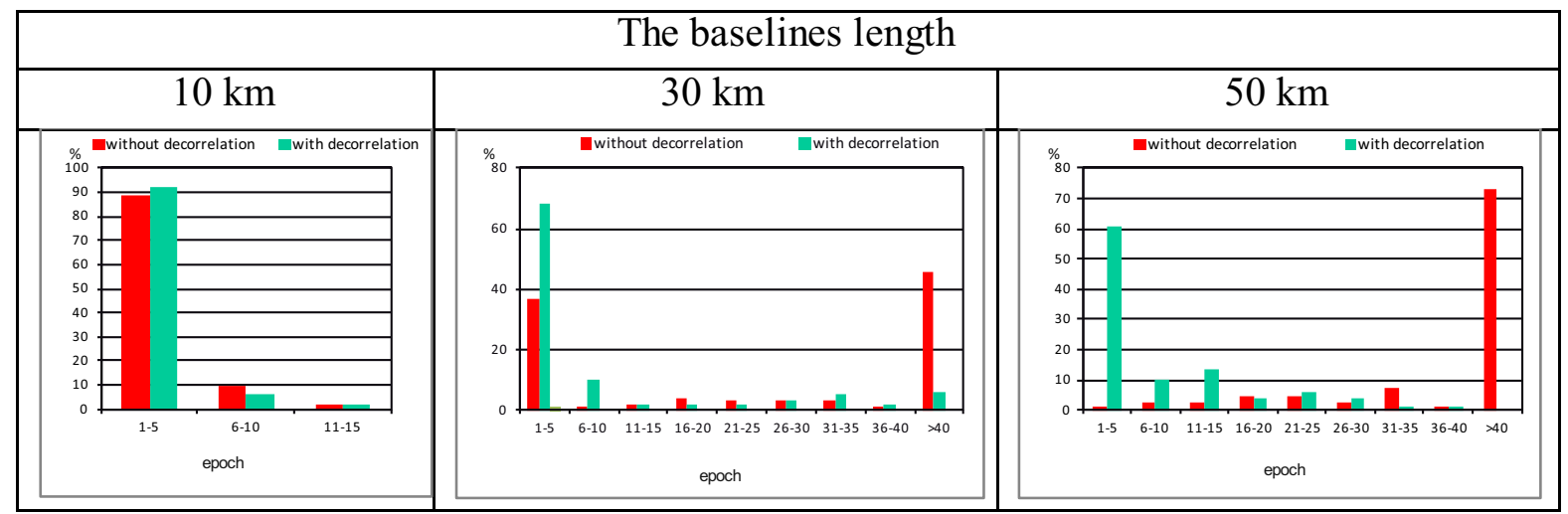

Fig. 3 The histograms of the correct stable solutions 


\section{CONCLUSIONS}

The integer decorrelation procedure can improve the efficiency of the MAFA method. The impact of the decorrelation procedure on the efficiency of the MAFA method depends on the baseline length. A longer baseline length corresponds to a higher impact of decorrelation on the efficiency of the MAFA method.

The MAFA method makes it possible to obtain good solutions from a single epoch only. In this case, the decorrelation procedure is performed on the basis of the biased estimator of the ambiguity covariance matrix. In order to derive a form of this matrix, a new coefficient $\mathrm{k}$ must be applied to obtain a positive definiteness of the covariance matrix.

\section{REFERENCES}

Cellmer S., Wielgosz P., Rzepecka Z. (2010) Modified ambiguity function approach for GPS carrier phase positioning. Journal of Geodesy, vol. 84, 264-275.

Cellmer S. (2011a) The real time precise positioning using MAFA method, The $8^{\text {th }}$ International Conference ENVIRONMENTAL ENGINEERING, selected papers, vol . III, Vilnius, s.1310-1314

Cellmer S. (2011b), A Graphic Representation of the Necessary Condition for the MAFA Method. Transactions on Geoscience and Remote Sensing, vol. PP Isssue: 99, 1-7.

Cellmer S. and Wielgosz P. (2011) "GNSS Carrier Phase Processing Using Modified Ambiguity Function Approach”, Florence, Italy, May 27-30 2009, EUREF Publication, Mitteilungen des Bundesamtes für Kartographie und Geodäsie (Sub mitted for publication), on line available

http://www.epncb.oma.be/_newsmails/papers/eurefsymposium2009/gnss_carrier_phase_ processing_using_modified_ambiguity_function.pdf

Chang X-W, Yang X, Zhou T. (2005) MLAMBDA: a modified LAMBDA method for integer least-squares estimation, Journal of Geodesy 79, 552-565.

Cocard M., Bourgon S. Kamali O., Collins P. (2008) A systematic investigation of optimal carrier-phase combinations for modernized triple-frequency GPS", Journal of Geodesy vol. 82, 555-564.

Dach R, Hugentobler U, Fridez P, Meindl M (2007) BERNESE GPS Software Version 5.0. Astronomical Institute, University of Berne.

Glenn J., Svedensen G. 2006. Some properties of decorrelation techniques in the ambiguity space GPS Solution vol. 10, 40-44.

Hassibi A. and Boyd S. (1998) Integer parameter estimation in linear models with application to GPS. IEEE Trans SignallProc 46, 2938-2952

Hofmann-Wellenhof B, Lichtenegger H, Wasle E. (2008) GNSS-Global Navigation Satellite Systems - GPS, GLONASS, Galileo \& more. Springer-Verlag Wien.

Han S, Rizos C. (1996) Improving the computational efficiency of the ambiguity function algorithm. Journal of Geodesy 70, 330-341.

Jonge P. de., Tiberius Ch. (1996) The LAMBDA method for integer ambiguity estimation: implementation aspects Delft Geodetic Computing Centre LGR Series.

Jung J. and Enge P. (2000) Optimization of Cascade Integer Resolution with Three Civil GPS Frequencies In Proc. ION GPS'2000, Salt Lake City, September

Leick A. (2004) GPS Satellite Surveying. $3^{\text {rd }}$ edition,John Wiley and Sons, Inc.

Liu L.T., Hsu H.T., Zhu Y.Z., Ou J.K. (1999) A new approach to GPS ambiguity decorrelation Journal of Geodesy vol. 73,478-490. 
Teunissen P J G. (1995) The least-squares ambiguity decorrelation adjustment: a method for fast GPS integer ambiguity estimation Journal of Geodesy 70, 65-82.

Teunissen P J G, and Kleusberg A. (1998) GPS for Geodesy, Springer - Verlag, Berlin Heidelberg New York.

Urquhart, L. (2009) An Analysis of Multi-Frequency Carrier Phase Linear Combinations for GNSS. Senior technical report, Department of Geodesy and Geomatics Engineering Technical Report No. 263, University of New Brunswick, Fredericton, New Brunswick, Canada.

Xu PL (2001) Random simulation and GPS decorrelation. Journal of Geodesy 75:408-423

Received: 2011-08-19,

Reviewed: 2011-10-11,

Accepted: 2011-10-13. 